\title{
Differential regulation of IL-22BP in Crohn's disease versus ulcerative colitis
}

\author{
Jérôme Martin ${ }^{1,2}$, Céline Bossard ${ }^{3}$, Arnaud Boureille $e^{4,5}$, Régis Josien ${ }^{1,2^{*}}$ \\ From 6th European Workshop on Immune-Mediated Inflammatory Diseases \\ Nice, France. 23-25 November 2011
}

\section{Introduction}

IL-22 is a newly described IL-10 cytokine family member. It mainly acts on epithelial cells and hepatocytes by interacting with a membrane receptor. IL-22 has been shown to have protective or deleterious effects on its targets cells depending on the context. IL-22 has been implicated in inflammatory bowel diseases (IBD) but its role still remains unclear. IL-22 is increased in Crohn's disease (CD) but not in ulcerative colitis (UC). Furthermore IL-22 appears to have beneficial effects in several murine models of IBD. IL-22BP is a soluble inhibitory receptor specific for IL-22 whose physiological role and regulation are mainly unknown during inflammatory conditions.

\section{Aims}

To assess the regulation of IL-22BP during IBD.

\section{Methods}

Colonic biopsies were obtained from patients with active $\mathrm{CD}$ or UC. Biopsies were made in inflammatory and noninflammatory mucosa for both conditions. Patients with polyps were used as healthy controls. IL-22BP mRNA expression was assessed by q-PCR and confirmed at the protein level by immunohistology, using a monoclonal $\mathrm{Ab}$ to IL-22BP. Informed consent was obtained from all the patients.

\section{Results}

No difference could be observed in the IL-22BP mRNA expression between the non inflammatory mucosa of $\mathrm{CD}$ or UC patients compared with healthy controls. In UC patients, IL-22BP was expressed at the same level in inflammatory or non inflammatory samples. In contrast, important up-regulation of IL-22BP mRNA expression was detected in the inflammatory mucosa of $\mathrm{CD}$ patients as compared to non inflammatory samples. This upregulation was confirmed at the protein level by immunostaining experiments. IL-22BP was mostly detected in the lamina propria of the colon. In UC patients, IL-22BP protein exhibited actually a diminished expression as compared to controls.

\section{Conclusion}

Taken together these results highlight a different profile of IL-22BP production during CD and UC. Up-regulation of IL-22BP during CD is probably concomitant to IL-22 up-regulation already described, suggesting an immunomodulatory function of IL-22BP specific to $C D$.

\section{Author details}

${ }^{1}$ INSERM U643, ITUN, Nantes, France. '2Laboratoire d'Immunologie, Nantes, France. ${ }^{3}$ Service d'Anatomo-Pathologie, Nantes, France. ${ }^{4}$ INSERM U913, Nantes, France. ${ }^{5}$ Service d'Hépato-Gastroentérologie, CHU Nantes, Nantes, France.

Published: 23 November 2011

doi:10.1186/1479-5876-9-S2-P11

Cite this article as: Martin et al.: Differential regulation of IL-22BP in Crohn's disease versus ulcerative colitis. Journal of Translational Medicine 2011 9(Suppl 2):P11. 